\title{
Existence and Exponential Stability of Periodic Solution of High-Order Hopfield Neural Network with Delays on Time Scales
}

\author{
Yongkun Li, Lili Zhao, and Ping Liu \\ Department of Mathematics, Yunnan University, Kunming, Yunnan 650091, China \\ Correspondence should be addressed to Yongkun Li, yklie@ynu.edu.cn
}

Received 17 April 2009; Accepted 3 August 2009

Recommended by Binggen Zhang

On time scales, by using the continuation theorem of coincidence degree theory and constructing some suitable Lyapunov functions, the periodicity and the exponential stability are investigated for a class of delayed high-order Hopfield neural networks (HHNNs), which are new and complement of previously known results. Finally, an example is given to show the effectiveness of the proposed method and results.

Copyright ( 2009 Yongkun Li et al. This is an open access article distributed under the Creative Commons Attribution License, which permits unrestricted use, distribution, and reproduction in any medium, provided the original work is properly cited.

\section{Introduction}

Consider the following HHNNs with time-varying delays:

$$
\begin{aligned}
\frac{d x_{i}(t)}{d t}= & -c_{i}(t) x_{i}(t)+\sum_{j=1}^{n} a_{i j}(t) f_{j}\left(x_{j}\left(t-\gamma_{i j}(t)\right)\right) \\
& +\sum_{j=1}^{n} \sum_{l=1}^{n} b_{i j l}(t) g_{j}\left(x_{j}\left(t-\sigma_{i j l}(t)\right)\right) g_{l}\left(x_{l}\left(t-v_{i j l}(t)\right)\right) \\
& +I_{i}(t), \quad i=1,2, \ldots, n,
\end{aligned}
$$

where $n$ corresponds to the number of units in a neural network, $x_{i}(t)$ corresponds to the state vector of the $i$ th unit at the time $t, c_{i}(t)$ represents the rate with which the $i$ th unit will reset its potential to the resting state in isolation when disconnected from the network and external inputs, $a_{i j}(t)$ and $b_{i j l}(t)$ are the first- and second-order connection weights of the 
neural network, $\gamma_{i j}(t) \geq 0, \sigma_{i j l}(t) \geq 0$, and $v_{i j l}(t) \geq 0$ correspond to the transmission delays, $I_{i}(t)$ denote the external inputs at time $t$, and $f_{j}$ and $g_{j}$ are the activation functions of signal transmission.

Due to the fact that high-order neural networks have stronger approximation property, faster convergence rate, greater storage capacity, and higher fault tolerance than lower-order neural networks, high-order neural networks have been the object of intensive analysis by numerous authors in the recent years. In particular, there have been extensive results on the problem of the existence and stability of equilibrium points and periodic solutions of HHNNs (1.1) in the literature. We refer the reader to [1-9] and the references cited therein. In fact, both continuous and discrete systems are very important in implementing and applications. The theory of calculus on time scales (see $[10,11]$ and references cited therein) was initiated by Stefan Hilger in his Ph.D. thesis in 1988 [12] in order to unify continuous and discrete analysis, and it has a tremendous potential for application and has recently received much attention since his foundational work. Therefore, it is meaningful to study that on time scales which can unify the continuous and discrete situations.

Our purpose of this paper is to consider the model

$$
\begin{aligned}
x_{i}^{\Delta}(t)= & -c_{i}(t) x_{i}(t)+\sum_{j=1}^{n} a_{i j}(t) f_{j}\left(x_{j}\left(t-\gamma_{i j}(t)\right)\right) \\
& +\sum_{j=1}^{n} \sum_{l=1}^{n} b_{i j l}(t) g_{j}\left(x_{j}\left(t-\sigma_{i j l}(t)\right)\right) g_{l}\left(x_{l}\left(t-v_{i j l}(t)\right)\right) \\
& +I_{i}(t), \quad i=1,2, \ldots, n, t \in \mathbb{T},
\end{aligned}
$$

where $\mathbb{T}$ is an $\omega$-periodic time scale which has the subspace topology inherited from the standard topology on $\mathbb{R}, I(t)=\left(I_{1}(t), I_{2}(t), \ldots, I_{n}(t)\right)^{T}$ is an input periodic vector function with period $\omega$; that is, there exists $\omega>0$ such that $I_{i}(t+\omega)=I_{i}(t)(i=1,2, \ldots, n)$ for all $t \in(0,+\infty) \cap \mathbb{T}$, and $x=\left(x_{1}, x_{2}, \ldots, x_{n}\right)^{T} \in \mathbb{R}^{n}, f(x)=\left(f_{1}\left(x_{1}\right), f_{2}\left(x_{2}\right), \ldots, f_{n}\left(x_{n}\right)\right)^{T}$, and $g(x)=$ $\left(g_{1}\left(x_{1}\right), g_{2}\left(x_{2}\right), \ldots, g_{n}\left(x_{n}\right)\right)^{T}$ is the activation function of the neurons.

System (1.2) is supplemented with initial values given by

$$
x_{i}(s)=\varphi_{i}(s), \quad s \in[-\theta, 0] \bigcap \mathbb{T}, i=1,2, \ldots, n,
$$

where $\varphi_{i}(\cdot)$ denotes continuous $\omega$-periodic function defined on $[-\theta, 0] \cap \mathbb{T}, \theta=$ $\max \{\gamma, \sigma, v\}, \gamma=\max _{1 \leq i, j \leq n}\left\{\gamma_{i j}^{+}\right\}, \sigma=\max _{1 \leq i, j, l \leq n}\left\{\sigma_{i j l}^{+}\right\}, v=\max _{1 \leq i, j, l \leq n}\left\{v_{i j l}^{+}\right\}, \gamma_{i j}^{+}=$ $\max _{t \in[0, \omega] \cap \mathbb{T}} \gamma_{i j}(t), \sigma_{i j l}^{+}=\max _{t \in[0, \omega] \cap \mathbb{T}} \sigma_{i j l}(t), v_{i j l}^{+}=\max _{t \in[0, \omega] \cap \mathbb{T}} v_{i j l}(t), i, j, l=1,2, \ldots, n$. Тo the best of our knowledge, this is the first paper to study the stability and existence of periodic solutions of (1.2).

Throughout this paper, we assume the following.

$\left(H_{1}\right)$ For $i, j, l=1,2, \ldots, n, c_{i}(t), a_{i j}(t), b_{i j l}(t), I_{i}(t), \gamma_{i j}(t), \sigma_{i j l}(t), v_{i j l}(t)$ are positive continuous periodic functions with period $\omega>0$, and $c_{i}(t)$ is regressive.

$\left(H_{2}\right)$ There exist positive constants $M_{j}, N_{j}, j=1,2, \ldots, n$ such that $\left|f_{j}(x)\right| \leq M_{j},\left|g_{j}(x)\right| \leq$ $N_{j}$ for $j=1,2, \ldots, n, x \in \mathbb{R}$. 
$\left(H_{3}\right)$ Functions $f_{j}(u), g_{j}(u)(j=1,2, \ldots, n)$ satisfy the Lipschitz condition; that is, there exist constants $L_{j}, H_{j}>0$ such that $\left|f_{j}\left(u_{1}\right)-f_{j}\left(u_{2}\right)\right| \leq L_{j}\left|u_{1}-u_{2}\right|,\left|g_{j}\left(u_{1}\right)-g_{j}\left(u_{2}\right)\right| \leq$ $H_{j}\left|u_{1}-u_{2}\right|, j=1,2, \ldots, n$.

\section{Preliminaries}

In this section, we will first recall some basic definitions and lemmas which are used in what follows.

Let $\mathbb{T}$ be a nonempty closed subset (time scale) of $\mathbb{R}$. The forward and backward jump operators $\sigma, \rho: \mathbb{T} \rightarrow \mathbb{T}$ and the graininess $\mu: \mathbb{T} \rightarrow \mathbb{R}^{+}$are defined, respectively, by

$$
\sigma(t)=\inf \{s \in \mathbb{T}: s>t\}, \quad \rho(t)=\sup \{s \in \mathbb{T}: s<t\}, \quad \mu(t)=\sigma(t)-t .
$$

A point $t \in \mathbb{T}$ is called left-dense if $t>\inf \mathbb{T}$ and $\rho(t)=t$, left-scattered if $\rho(t)<t$, right-dense if $t<\sup \mathbb{T}$ and $\sigma(t)=t$, and right-scattered if $\sigma(t)>t$. If $\mathbb{T}$ has a left-scattered maximum $m$, then $\mathbb{T}^{k}=\mathbb{T} \backslash\{m\}$; otherwise $\mathbb{T}^{k}=\mathbb{T}$. If $\mathbb{T}$ has a right-scattered minimum $m$, then $\mathbb{T}_{k}=\mathbb{T} \backslash\{m\}$; otherwise $\mathbb{T}_{k}=\mathbb{T}$.

A function $f: \mathbb{T} \rightarrow \mathbb{R}$ is right-dense continuous provided that it is continuous at right-dense point in $\mathbb{T}$ and its left-side limits exist at left-dense points in $\mathbb{T}$. If $f$ is continuous at each right-dense point and each left-dense point, then $f$ is said to be continuous function on $\mathbb{T}$.

For $y: \mathbb{T} \rightarrow \mathbb{R}$ and $t \in \mathbb{T}^{k}$, we define the delta derivative of $y(t), y^{\Delta}(t)$ to be the number (if it exists) with the property that for a given $\varepsilon>0$, there exists a neighborhood $U$ of $t$ such that

$$
\left|[y(\sigma(t))-y(s)]-y^{\Delta}(t)[\sigma(t)-s]\right|<\varepsilon|\sigma(t)-s|
$$

for all $s \in U$.

If $y$ is continuous, then $y$ is right-dense continuous, and if $y$ is delta differentiable at $t$, then $y$ is continuous at $t$.

A function $r: \mathbb{T} \rightarrow \mathbb{R}$ is called regressive if

$$
1+\mu(t) r(t) \neq 0
$$

for all $t \in \mathbb{T}^{k}$.

If $r$ is regressive function, then the generalized exponential function $e_{r}$ is defined by

$$
e_{r}(t, s)=\exp \left\{\int_{s}^{t} \xi_{\mu(\tau)}(r(\tau)) \Delta \tau\right\} \quad \text { for } s, t \in \mathbb{T},
$$

with the cylinder transformation

$$
\xi_{h}(z)= \begin{cases}\frac{\log (1+h z)}{h}, & \text { if } h \neq 0 \\ z, & \text { if } h=0\end{cases}
$$


Let $p, q: \mathbb{T} \rightarrow \mathbb{R}$ be two regressive functions, we define

$$
p \oplus q:=p+q+\mu p q, \quad \ominus p:=-\frac{p}{1+\mu p}, \quad p \ominus q:=p \oplus(\ominus q)
$$

Then the generalized exponential function has the following properties.

Lemma 2.1 (see [10]). Assume that $p, q: \mathbb{T} \rightarrow \mathbb{R}$ are two regressive functions, then

(i) $e_{0}(t, s) \equiv 1$ and $e_{p}(t, t) \equiv 1$;

(ii) $e_{p}(\sigma(t), s)=(1+\mu(t) p(t)) e_{p}(t, s)$;

(iii) $e_{p}(t, \sigma(s))=e_{p}(t, s) /(1+\mu(s) p(s))$;

(iv) $1 / e_{p}(t, s)=e_{\ominus p}(t, s)$;

(v) $e_{p}(t, s)=1 / e_{p}(s, t)=e_{\ominus p}(s, t)$;

(vi) $e_{p}(t, s) e_{p}(s, r)=e_{p}(t, r)$;

(vii) $e_{p}(t, s) e_{q}(t, s)=e_{p \oplus q}(t, s)$;

(viii) $e_{p}(t, s) / e_{q}(t, s)=e_{p \ominus q}(t, s)$.

Lemma 2.2 (see [10]). Assume that $f, g: \mathbb{T} \rightarrow \mathbb{R}$ are delta differentiable at $t \in \mathbb{T}^{k}$, then

$$
(f g)^{\Delta}(t)=f^{\Delta}(t) g(t)+f(\sigma(t)) g^{\Delta}(t)=f(t) g^{\Delta}(t)+f^{\Delta}(t) g(\sigma(t)) .
$$

Let $y$ be right-dense continuous. If $Y^{\Delta}(t)=y(t)$, then one defines the delta integral by

$$
\int_{a}^{t} y(s) \Delta s=Y(t)-Y(a)
$$

Lemma 2.3. If $a, b \in \mathbb{T}, \alpha, \beta \in \mathbb{R}$ and $f, g \in C(\mathbb{T}, \mathbb{R})$, then

(1) $\int_{a}^{b}[\alpha f(t)+\beta g(t)] \Delta t=\alpha \int_{a}^{b} f(t) \Delta t+\beta \int_{a}^{b} g(t) \Delta t ;$

(2) If $f(t) \geq 0$ for all $a \leq t<b$, then $\int_{a}^{b} f(t) \Delta t \geq 0$;

(3) If $|f(t)| \leq g(t)$ on $[a, b):=\{t \in \mathbb{T}: a \leq t<b\}$, then $\left|\int_{a}^{b} f(t) \Delta t\right| \leq \int_{a}^{b} g(t) \Delta t$.

In this paper, one assumes that $\bar{k}=\min \{[0,+\infty) \cap \mathbb{T}\}$. Clearly, from Lemma 2.3, one can obtain Lemma 2.4.

Lemma 2.4. If $f, g \in C(\mathbb{T}, \mathbb{R})$, and $f(t) \leq g(t)$ on $t \in[\bar{k}, \bar{k}+\omega)$, then $\int_{\bar{k}}^{\bar{k}+\omega} f(t) \Delta t \leq \int_{\bar{k}}^{\bar{k}+\omega} g(t) \Delta t$.

In the proof of our main result, one will use the following three lemmas which can be found in $[13,14]$.

Lemma 2.5 (see [13]). Let $t_{1}, t_{2} \in[\bar{k}, \bar{k}+\omega] \cap \mathbb{T}$, and $t \in \mathbb{T}$. If $f: \mathbb{T} \rightarrow \mathbb{R}$ is w-periodic, then $f(t) \leq f\left(t_{1}\right)+\int_{\bar{k}}^{\bar{k}+\omega}\left|f^{\Delta}(s)\right| \Delta s$, and $f(t) \geq f\left(t_{2}\right)-\int_{\bar{k}}^{\bar{k}+\omega}\left|f^{\Delta}(s)\right| \Delta s$. 
Lemma 2.6 ([14], Cauchy-Schwarz inequality on time scales). Let $a, b \in \mathbb{T}$. For $r d$-continuous functions $f, g:[a, b] \rightarrow \mathbb{R}$, one has $\left(\int_{a}^{b}|f(t) g(t)| \Delta t\right)^{2} \leq \int_{a}^{b}|f(t)|^{2} \Delta t \int_{a}^{b}|g(t)|^{2} \Delta t$.

Lemma 2.7. Let $r: \mathbb{T} \rightarrow \mathbb{R}$ be right-dense continuous and regressive. $a \in \mathbb{T}$, and $y_{a} \in \mathbb{R}$. The unique solution of the initial value problem

$$
y^{\Delta}(t)=r(t) y(t)+h(t), \quad y(a)=y_{a}
$$

is given by

$$
y(t)=e_{r}(t, a) y_{a}+\int_{a}^{t} e_{r}(t, \sigma(s)) h(s) \Delta s
$$

For the sake of convenience, one introduces the following notations:

$$
\begin{aligned}
\overline{c_{i}} & =\frac{1}{\omega} \int_{\bar{k}}^{\bar{k}+\omega} c_{i}(t) \Delta t, \quad \overline{a_{i j}}=\frac{1}{\omega} \int_{\bar{k}}^{\bar{k}+\omega} a_{i j}(t) \Delta t, \quad \overline{I_{i}}=\frac{1}{\omega} \int_{\bar{k}}^{\bar{k}+\omega} I_{i}(t) \Delta t, \\
\overline{b_{i j l}} & =\frac{1}{\omega} \int_{\bar{k}}^{\bar{k}+\omega} b_{i j l}(t) \Delta t, \quad c_{i}^{+}=\max _{t \in[\bar{k}, \bar{k}+\omega] \cap \mathbb{T}}\left|c_{i}(t)\right|, \quad c_{i}^{-}=\min _{t \in[\bar{k}, \bar{k}+\omega] \cap \mathbb{T}}\left|c_{i}(t)\right|, \\
a_{i j}^{+}= & \max _{t \in[\bar{k}, \bar{k}+\omega] \cap \mathbb{T}}\left|a_{i j}(t)\right|, \quad b_{i j l}^{+}=\max _{t \in[\bar{k}, \bar{k}+\omega] \cap \mathbb{T}}\left|b_{i j l}(t)\right|, \quad I_{i}^{+}=\max _{t \in[\bar{k}, \bar{k}+\omega] \cap \mathbb{T}}\left|I_{i}(t)\right|, \\
A_{i}(t)= & -c_{i}(t) x_{i}(t)+\sum_{j=1}^{n} a_{i j}(t) f_{j}\left(x_{j}\left(t-\gamma_{i j}(t)\right)\right) \\
& +\sum_{j=1}^{n} \sum_{l=1}^{n} b_{i j l}(t) g_{j}\left(x_{j}\left(t-\sigma_{i j l}(t)\right)\right) g_{l}\left(x_{l}\left(t-v_{i j l}(t)\right)\right)+I_{i}(t), \quad i, j, l=1,2, \ldots n .
\end{aligned}
$$

Obliviously, for system (1.2), finding the periodic solutions is equivalent to finding those of the following boundary-value problem:

$$
\begin{aligned}
x_{i}^{\Delta}(t)= & -c_{i}(t) x_{i}(t)+\sum_{j=1}^{n} a_{i j}(t) f_{j}\left(x_{j}\left(t-\gamma_{i j}(t)\right)\right) \\
& +\sum_{j=1}^{n} \sum_{l=1}^{n} b_{i j l}(t) g_{j}\left(x_{j}\left(t-\sigma_{i j l}(t)\right)\right) g_{l}\left(x_{l}\left(t-v_{i j l}(t)\right)\right)+I_{i}(t), \\
& t \in[\bar{k}, \bar{k}+\omega] \cap \mathbb{T}, \quad i=1,2, \ldots, n, \\
x_{i}(\bar{k})= & x_{i}(\bar{k}+\omega), \quad i=1,2, \ldots, n .
\end{aligned}
$$

Now, one states Mawhin's continuous theorem [15]. 
Theorem 2.8. Let $X$ and $Z$ be two Banach spaces and let $L$ be a Fredholm mapping of index zero. let $\boldsymbol{\Omega} \subset \mathbb{X}$ be an open bounded set and let $N: \overline{\boldsymbol{\Omega}} \rightarrow \mathbb{Z}$ be a continuous operator which is $L$-compact on $\overline{\mathbf{\Omega}}$. Assume that

(1) for each $\lambda \in(0,1), x \in \partial \boldsymbol{\Omega} \cap \operatorname{Dom} L, L x \neq \lambda N x$;

(2) for each $x \in \partial \boldsymbol{\Omega} \cap \operatorname{Ker} L, Q N x \neq 0$;

(3) $\operatorname{deg}(J N Q x, \Omega \cap \operatorname{Ker} L, 0) \neq 0$, where $J Q N: \operatorname{Ker} L \rightarrow \operatorname{Ker} L$.

Then $L x=N x$ has at least one solution in $\overline{\mathbf{\Omega}} \cap \operatorname{Dom} L$.

In order to apply Theorem 2.8 to system (2.12), we first define

$$
\begin{aligned}
X & =Z=\left\{x=\left(x_{1}, x_{2}, \ldots, x_{n}\right)^{T} \in C\left(\mathbb{T}, \mathbb{R}^{n}\right): x(t+\omega)=x(t), t \in \mathbb{T}\right\}, \\
\|x\| & =\left\|\left(x_{1}, x_{2}, \ldots, x_{n}\right)^{T}\right\|=\sum_{i=1}^{n} \max _{t \in[\bar{k}, \bar{k}+\omega] \cap \mathbb{T}}\left|x_{i}(t)\right|
\end{aligned}
$$

for any $x \in X($ or $Z)$. Then $X$ and $Z$ are Banach spaces with the norm $\|\cdot\|$. Let

$$
\begin{gathered}
N x=\left(A_{1}(t), A_{2}(t), \ldots, A_{n}(t)\right)^{T}, \quad x \in X \\
L x=\left(x_{1}^{\Delta}, x_{2}^{\Delta}, \ldots, x_{n}^{\Delta}\right)^{T} \\
P x=\left(\frac{1}{\omega} \int_{\bar{k}}^{\bar{k}+\omega} x_{1}(t) \Delta t, \frac{1}{\omega} \int_{\bar{k}}^{\bar{k}+\omega} x_{2}(t) \Delta t, \ldots, \frac{1}{\omega} \int_{\bar{k}}^{\bar{k}+\omega} x_{n}(t) \Delta t\right)^{T}, \\
Q z=\left(\frac{1}{\omega} \int_{\bar{k}}^{\bar{k}+\omega} z_{1}(t) \Delta t, \frac{1}{\omega} \int_{\bar{k}}^{\bar{k}+\omega} z_{2}(t) \Delta t, \ldots, \frac{1}{\omega} \int_{\bar{k}}^{\bar{k}+\omega} z_{n}(t) \Delta t\right)^{T}, \quad z \in Z .
\end{gathered}
$$

Then it follows that

$$
\begin{aligned}
\text { Ker } L & =\left\{\left(x_{1}, x_{2}, \ldots, x_{n}\right)^{T} \in \mathbb{X}:\left(x_{1}, x_{2}, \ldots, x_{n}\right)^{T}=\left(w_{1}(t), w_{2}(t), \ldots, w_{n}(t)\right)^{T} \in \mathbb{R}^{n}, t \in \mathbb{T}\right\}, \\
\operatorname{Im} L & =\left\{z \in \mathbb{Z}:\left(\frac{1}{\omega} \int_{\bar{k}}^{\bar{k}+\omega} z_{1}(t) \Delta t, \frac{1}{\omega} \int_{\bar{k}}^{\bar{k}+\omega} z_{2}(t) \Delta t, \ldots, \frac{1}{\omega} \int_{\bar{k}}^{\bar{k}+\omega} z_{n}(t) \Delta t\right)^{T}=0\right\}
\end{aligned}
$$

is closed in $Z, \operatorname{dim} \operatorname{Ker} L=n=\operatorname{codim} \operatorname{Im} L$. It is not difficult to show that $P$ and $Q$ are continuous and satisfy $\operatorname{Im} P=\operatorname{Ker} L, \operatorname{Im} L=\operatorname{Ker} Q=\operatorname{Im}(I-Q)$. It is easy to see that $\operatorname{Im} L$ is closed in $Z$, which leads to the following lemma.

Lemma 2.9. Let $L$ and $N$ be defined by (2.14) and (2.15), respectively, then $L$ is a Fredholm operator of index zero. 
Lemma 2.10. Let $L$ and $N$ be defined by (2.14) and (2.15), respectively, suppose that $\Omega$ is an open bounded subset of Dom $L$, then $N$ is L-compact on $\bar{\Omega}$.

Proof. Through an easy computation, we find that the inverse $K_{P}: \operatorname{Im} L \rightarrow \operatorname{Ker} P \cap \operatorname{Dom} L$ of $L_{P}$ has the form

$$
\left(K_{P} z\right)(t)=\int_{\bar{k}}^{t} z(s) \Delta s-\frac{1}{\omega} \int_{\bar{k}}^{\bar{k}+\omega} \int_{\bar{k}}^{t} z(s) \Delta s \Delta t
$$

Thus, the expression of $Q N x$ is

$$
Q N x=\left(\frac{1}{\omega} \int_{\bar{k}}^{\bar{k}+\omega} A_{1}(t) \Delta t, \frac{1}{\omega} \int_{\bar{k}}^{\bar{k}+\omega} A_{2}(t) \Delta t, \ldots, \frac{1}{\omega} \int_{\bar{k}}^{\bar{k}+\omega} A_{n}(t) \Delta t\right)^{T},
$$

and then

$$
\begin{aligned}
K_{P}(I-Q) N x= & \left(\begin{array}{c}
\int_{\bar{k}}^{t} A_{1}(s) \Delta s \\
\vdots \\
\int_{\bar{k}}^{t} A_{n}(s) \Delta s
\end{array}\right) \\
& -\left(\begin{array}{c}
\frac{1}{\omega} \int_{\bar{k}}^{\bar{k}+\omega} \int_{\bar{k}}^{t} A_{1}(s) \Delta s \Delta t \\
\vdots \\
\frac{1}{\omega} \int_{\bar{k}}^{\bar{k}+\omega} \int_{\bar{k}}^{t} A_{n}(s) \Delta s \Delta t
\end{array}\right) \\
& -\left(\begin{array}{l}
\left(\frac{t-\bar{k}}{\omega}-\frac{1}{(\omega)^{2}} \int_{\bar{k}}^{\bar{k}+\omega}(t-\bar{k}) \Delta t\right) \int_{\bar{k}}^{\bar{k}+\omega} A_{1}(s) \Delta s \\
\left(\frac{t-\bar{k}}{\omega}-\frac{1}{(\omega)^{2}} \int_{\bar{k}}^{\bar{k}+\omega}(t-\bar{k}) \Delta t\right) \int_{\bar{k}}^{\bar{k}+\omega} A_{n}(s) \Delta s
\end{array}\right) .
\end{aligned}
$$

Thus, $Q N$ and $K_{P}(I-Q) N$ are continuous. Since $X$ is a Banach space, it is not difficult to show that $\overline{K_{P}(I-Q) N(\bar{\Omega})}$ is compact. Moreover, $Q N(\bar{\Omega})$ is bounded. Thus, $N$ is $L$-compact on $\bar{\Omega}$ for any open bounded set $\Omega \subset$ X. The proof of Lemma 2.10 is completed.

\section{Existence of Periodic Solution}

In this section, we study the existence of periodic solution of (1.2) based on Mawhin's continuation theorem. 
Theorem 3.1. Assume that $\left(H_{1}\right)-\left(H_{2}\right)$ hold, then system (1.2) has at least one w-periodic solution.

Proof. Based on the Lemma 2.9 and Lemma 2.10, now, what we need to do is just to search for an appropriate open, bounded subset $\Omega$ for the application of the continuation theorem. Corresponding to the operator equation $L x=\lambda N x, \lambda \in(0,1)$, we have

$$
\begin{aligned}
& x_{i}^{\Delta}(t)=\lambda[-c_{i}(t) x_{i}(t)+\sum_{j=1}^{n} a_{i j}(t) f_{j}\left(x_{j}\left(t-\gamma_{i j}(t)\right)\right) \\
&+\sum_{j=1}^{n} \sum_{l=1}^{n} b_{i j l}(t) g_{j}\left(x_{j}\left(t-\sigma_{i j l}(t)\right)\right) g_{l}\left(x_{l}\left(t-v_{i j l}(t)\right)\right) \\
&\left.+I_{i}(t), t \in \mathbb{T} \bigcap[\bar{k}, \bar{k}+\omega]\right], \quad i=1,2, \ldots, n, \\
& x_{i}(\bar{k})=x_{i}(\bar{k}+\omega), \quad i=1,2, \ldots, n .
\end{aligned}
$$

For the sake of convenience, defined $\|x\|_{2}$ by

$$
\|x\|_{2}=\left(\int_{\bar{k}}^{\bar{k}+\omega}|x(t)|^{2} \Delta t\right)^{1 / 2}
$$

for $x \in C(\mathbb{T}, \mathbb{R})$. Suppose that $\left(x_{1}(t), x_{2}(t), \ldots, x_{n}(t)\right)^{T} \in X$ is a solution of system (3.1) for a certain $\lambda \in(0,1)$. Integrating $(3.1)$ over $[\bar{k}, \bar{k}+\omega]$, we obtain

$$
\int_{\bar{k}}^{\bar{k}+\omega} A_{i}(t) \Delta t=0
$$

Hence

$$
\begin{aligned}
& \int_{\bar{k}}^{\bar{k}+\omega} c_{i}(s) x_{i}(s) \Delta s \\
& =\left[\int_{\bar{k}}^{\bar{k}+\omega} \sum_{j=1}^{n} a_{i j}(t) f_{j}\left(x_{j}\left(t-\gamma_{i j}(t)\right)\right)+\sum_{j=1}^{n} \sum_{l=1}^{n} b_{i j l}(t) g_{j}\left(x_{j}\left(t-\sigma_{i j l}(t)\right)\right) g_{l}\left(x_{l}\left(t-v_{i j l}(t)\right)\right)+I_{i}(t)\right] \Delta t, \\
& i=1,2, \ldots, n .
\end{aligned}
$$


Let $\zeta_{i}, \eta_{i} \in \mathbb{T} \cap[\bar{k}, \bar{k}+\omega]$, such that $x_{i}\left(\zeta_{i}\right)=\inf _{t \in \mathbb{T} \cap[\bar{k}, \bar{k}+\omega]} x_{i}(t), x_{i}\left(\eta_{i}\right)=\sup _{t \in \mathbb{T} \cap[\bar{k}, \bar{k}+\omega]} x_{i}(t)$. Then by (3.4) and Lemma 2.4, we have

$$
\begin{aligned}
& \omega \overline{c_{i}} x_{i}\left(\zeta_{i}\right) \leq \int_{\bar{k}}^{\bar{k}+\omega}\left[\mid \sum_{j=1}^{n} a_{i j}(s) f_{j}\left(x_{j}\left(s-\gamma_{i j}(s)\right)\right)\right.\left.\quad+\sum_{j=1}^{n} \sum_{l=1}^{n} b_{i j l}(s) g_{j}\left(x_{j}\left(s-\sigma_{i j l}(s)\right)\right) g_{l}\left(x_{l}\left(s-v_{i j l}(s)\right)\right)+I_{i}(s) \mid\right] \Delta s \\
& \leq \int_{\bar{k}}^{\bar{k}+\omega} \sum_{j=1}^{n}\left|a_{i j}(s)\right|\left|f_{j}\left(x_{j}\left(s-\gamma_{i j}(s)\right)\right)\right| \Delta s \\
& \quad+\int_{\bar{k}}^{\bar{k}+\omega} \sum_{j=1}^{n} \sum_{l=1}^{n}\left|b_{i j l}(s)\left\|g_{j}\left(x_{j}\left(s-\sigma_{i j l}(s)\right)\right)\right\| g_{l}\left(x_{l}\left(s-v_{i j l}(s)\right)\right)\right| \Delta s+\int_{\bar{k}}^{\bar{k}+\omega} I_{i}(s) \Delta s \\
& \leq \omega\left[\sum_{j=1}^{n} a_{i j}^{+} M_{j}+\sum_{j=1}^{n} \sum_{l=1}^{n} b_{i j l}^{+} N_{j} N_{l}+I_{i}^{+}\right], \quad i=1,2, \ldots, n .
\end{aligned}
$$

Hence $x_{i}\left(\zeta_{i}\right) \leq\left(1 / \overline{c_{i}}\right)\left\{\left[\sum_{j=1}^{n} a_{i j}^{+} M_{j}+\sum_{j=1}^{n} \sum_{l=1}^{n} b_{i j l}^{+} N_{j} N_{l}+I_{i}^{+}\right]\right\}:=B_{i}, i=1,2, \ldots, n$. By (3.4) and Lemma 2.4, we can also have

$$
\begin{aligned}
\omega \overline{c_{i}} x_{i}\left(\eta_{i}\right) \geq & -\int_{\bar{k}}^{\bar{k}+\omega}\left[\mid \sum_{j=1}^{n} a_{i j}(s) f_{j}\left(x_{j}\left(s-\gamma_{i j}(s)\right)\right)\right. \\
& \left.+\sum_{j=1}^{n} \sum_{l=1}^{n} b_{i j l}(s) g_{j}\left(x_{j}\left(s-\sigma_{i j l}(s)\right)\right) g_{l}\left(x_{l}\left(s-v_{i j l}(s)\right)\right)+I_{i}(s) \mid\right] \Delta s \\
\geq & -\int_{\bar{k}}^{\bar{k}+\omega} \sum_{j=1}^{n}\left|a_{i j}(s)\right|\left|f_{j}\left(x_{j}\left(s-\gamma_{i j}(s)\right)\right)\right| \Delta s \\
& -\int_{\bar{k}}^{\bar{k}+\omega} \sum_{j=1}^{n} \sum_{l=1}^{n}\left|b_{i j l}(s)\right|\left|g_{j}\left(x_{j}\left(s-\sigma_{i j l}(s)\right)\right)\right|\left|g_{l}\left(x_{l}\left(s-v_{i j l}(s)\right)\right)\right| \Delta s-\int_{\bar{k}}^{\bar{k}+\omega}\left|I_{i}(s)\right| \Delta s \\
\geq & -\omega\left[\sum_{j=1}^{n} a_{i j}^{+} M_{j}+\sum_{j=1}^{n} \sum_{l=1}^{n} b_{i j l}^{+} N_{j} N_{l}+I_{i}^{+}\right], \quad i=1,2, \ldots, n .
\end{aligned}
$$


Hence $x_{i}\left(\eta_{i}\right) \geq\left(-1 / \overline{c_{i}}\right)\left\{\left[\sum_{j=1}^{n} a_{i j}^{+} M_{j}+\sum_{j=1}^{n} \sum_{l=1}^{n} b_{i j l}^{+} N_{j} N_{l}+I_{i}^{+}\right]\right\}=-B_{i}, i=1,2, \ldots, n$. From (3.1), (3.4), and Lemma 2.6, we have

$$
\begin{aligned}
\int_{\bar{k}}^{\bar{k}+\omega}\left|x_{i}^{\Delta}(t)\right| \Delta t & \\
\leq & \int_{\bar{k}}^{\bar{k}+\omega}\left|c_{i}(t)\right|\left|x_{i}(t)\right| \Delta t+\int_{\bar{k}}^{\bar{k}+\omega} \sum_{j=1}^{n}\left|a_{i j}(t)\right|\left|f_{j}\left(x_{j}\left(t-\gamma_{i j}(t)\right)\right)\right| \Delta t \\
& +\int_{\bar{k}}^{\bar{k}+\omega} \sum_{j=1}^{n} \sum_{l=1}^{n}\left|b_{i j l}(t)\right|\left|g_{j}\left(x_{j}\left(t-\sigma_{i j l}(t)\right)\right)\right|\left|g_{l}\left(x_{l}\left(t-v_{i j l}(t)\right)\right)\right| \Delta t+\int_{\bar{k}}^{\bar{k}+\omega}\left|I_{i}(t)\right| \Delta t \\
\leq & \left(\int_{\bar{k}}^{\bar{k}+\omega}\left|c_{i}(t)\right|^{2} \Delta t\right)^{1 / 2}\left(\int_{\bar{k}}^{\bar{k}+\omega}\left|x_{i}(t)\right|^{2} \Delta t\right)^{1 / 2} \\
& +\sum_{j=1}^{n}\left(\int_{\bar{k}}^{\bar{k}+\omega}\left|a_{i j}(t)\right|^{2} \Delta t\right)^{1 / 2}\left(\int_{\bar{k}}^{\bar{k}+\omega}\left|f_{j}\left(x_{j}\left(t-\gamma_{i j}(t)\right)\right)\right|^{2} \Delta t\right)^{1 / 2} \\
& +\sum_{j=1}^{n} \sum_{l=1}^{n} b_{i j l}^{+}\left(\int_{\bar{k}}^{\bar{k}+\omega}\left|g_{j}\left(x_{j}\left(t-\sigma_{i j l}(t)\right)\right)\right|^{2} \Delta t\right)^{1 / 2}\left(\int_{\bar{k}}^{\bar{k}+\omega}\left|g_{l}\left(x_{l}\left(t-\sigma_{i j l}(t)\right)\right)\right|^{2} \Delta t\right)^{1 / 2}+I_{i}^{+} \omega \\
\leq & (\omega)^{1 / 2} c_{i}^{+}\left\|x_{i}\right\|_{2}+\sum_{j=1}^{n} \omega a_{i j}^{+} M_{j}+\sum_{j=1}^{n} \sum_{l=1}^{n}(\omega) b_{i j l}^{+} N_{j} N_{l}+I_{i}^{+} \omega .
\end{aligned}
$$

From Lemma 2.7 and (3.1), for $i=1,2, \ldots, n$, we can obtain

$$
\begin{aligned}
& x_{i}(t)= e_{-\lambda c_{i}(t)}(t, \bar{k}) x_{i}(\bar{k}) \\
&+\int_{\bar{k}}^{t} \lambda e_{-\lambda c_{i}(t)}(t, \sigma(s))\left[\sum_{j=1}^{n} a_{i j}(s) f_{j}\left(x_{j}\left(s-\gamma_{i j}(s)\right)\right)\right. \\
& \\
&\left.\quad+\sum_{j=1}^{n} \sum_{l=1}^{n} b_{i j l}(s) g_{j}\left(x_{j}\left(s-\sigma_{i j l}(s)\right)\right) g_{l}\left(x_{l}\left(s-v_{i j l}(s)\right)\right)+I_{i}(s)\right] \Delta s .
\end{aligned}
$$


Hence

$$
\begin{aligned}
\left|x_{i}(t)\right| \leq & \left|x_{i}(\bar{k})\right|+\sum_{j=1}^{n} \int_{\bar{k}}^{\bar{k}+\omega}\left|a_{i j}(s)\right|\left|f_{j}\left(x_{j}\left(s-\gamma_{i j}(s)\right)\right)\right| \Delta s \\
& +\sum_{j=1}^{n} \sum_{l=1}^{n} \int_{\bar{k}}^{\bar{k}+\omega}\left|b_{i j l}(s)\right|\left|g_{j}\left(x_{j}\left(s-\sigma_{i j l}(s)\right)\right)\right|\left|g_{l}\left(x_{l}\left(s-v_{i j l}(s)\right)\right)\right| \Delta s \\
& +\int_{\bar{k}}^{\bar{k}+\omega}\left|I_{i}(s)\right| \Delta s \\
\leq & \left|x_{i}(\bar{k})\right|+\sum_{j=1}^{n} \overline{a_{i j}} N_{j} \omega+\sum_{j=1}^{n} \sum_{l=1}^{n} \overline{b_{i j l}} N_{j} N_{l} \omega+\overline{I_{i}} \omega \\
= & : u_{i}, \quad i=1,2, \ldots, n,
\end{aligned}
$$

that is,

$$
\left\|x_{i}\right\|_{2}=\left(\int_{\bar{k}}^{\bar{k}+\omega}\left|x_{i}(t)\right|^{2} \Delta t\right)^{1 / 2} \leq u_{i}(\omega)^{1 / 2}, \quad i=1,2, \ldots, n
$$

Substituting (3.10) into (3.7), we have

$$
\int_{\bar{k}}^{\bar{k}+\omega}\left|x_{i}^{\Delta}(t)\right| \Delta t \leq \omega c_{i}^{+} u_{i}+\sum_{j=1}^{n} \omega a_{i j}^{+} M_{j}+\sum_{j=1}^{n} \sum_{l=1}^{n}(\omega) b_{i j l}^{+} N_{j} N_{l}+I_{i}^{+} \omega, \quad i=1,2, \ldots, n
$$

From Lemma 2.5, we have

$$
\begin{aligned}
& x_{i}(t) \leq x_{i}\left(\zeta_{i}\right)+\int_{\bar{k}}^{\bar{k}+\omega}\left|x_{i}^{\Delta}(t)\right| \Delta t, \quad i=1,2, \ldots, n, \\
& x_{i}(t) \geq x_{i}\left(\eta_{i}\right)-\int_{\bar{k}}^{\bar{k}+\omega}\left|x_{i}^{\Delta}(t)\right| \Delta t, \quad i=1,2, \ldots, n .
\end{aligned}
$$

From (3.5), (3.6) and (3.11), there exist positive constants $\xi_{i}(i=1,2, \ldots, n)$ such that for $t \in[\bar{k}, \bar{k}+\omega] \cap \mathbb{T},\left|x_{i}(t)\right| \leq \xi_{i}, i=1,2, \ldots, n$. Clearly, $\xi_{i}(i=1,2, \ldots, n)$ is independent of $\lambda$. Denote $H^{*}=\sum_{i=1}^{n} \xi_{i}+C$, where $C>0$ is taken sufficiently large so that

$$
\min _{1 \leq i \leq n} \overline{\bar{C}_{i}} H^{*}>n \max _{1 \leq i \leq n}\left(\left|\overline{I_{i}}\right|+\sum_{j=1}^{n}\left|\overline{a_{i j}}\right| M_{j}+\sum_{j=1}^{n} \sum_{l=1}^{n}\left|\overline{b_{i j l}}\right| N_{j} N_{l}\right)
$$


Now we take $\Omega=\left\{\left(x_{1}(t), x_{2}(t), \ldots, x_{n}(t)\right)^{T}:\left\|\left(x_{1}(t), x_{2}(t), \ldots, x_{n}(t)\right)^{T}\right\|<H^{*}\right\}$. Thus (1) of Theorem 2.8 is satisfied. When $\left(x_{1}(t), x_{2}(t), \ldots, x_{n}(t)\right)^{T} \in \partial \Omega \bigcap \mathbb{R}^{n},\left(x_{1}(t)\right.$, $\left.x_{2}(t), \ldots, x_{n}(t)\right)^{T}$ is a constant vector in $\mathbb{R}^{n}$ with $\left|x_{1}\right|+\left|x_{2}\right|+\cdots+\left|x_{n}\right|=H^{*}$, then

$$
\begin{aligned}
Q N\left(x_{1}(t), x_{2}(t), \ldots, x_{n}(t)\right)^{T}=( & -\overline{c_{i}} x_{i}(t)+\sum_{j=1}^{n} \overline{a_{i j}} f_{j}\left(x_{j}\left(t-\gamma_{i j}(t)\right)\right) \\
& \left.+\sum_{j=1}^{n} \sum_{l=1}^{n} \overline{b_{i j l}} g_{j}\left(x_{j}\left(t-\sigma_{i j l}(t)\right)\right) g_{l}\left(x_{l}\left(t-v_{i j l}(t)\right)\right)+\overline{I_{i}}\right)_{n \times 1} .
\end{aligned}
$$

Therefore

$$
\begin{aligned}
\left\|Q N\left(x_{1}(t), x_{2}(t), \ldots, x_{n}(t)\right)^{T}\right\|= & \sum_{i=1}^{n} \mid \overline{c_{i}} x_{i}(t)-\sum_{j=1}^{n} \overline{a_{i j}} f_{j}\left(x_{j}\left(t-\gamma_{i j}(t)\right)\right) \\
& -\sum_{j=1}^{n} \sum_{l=1}^{n} \overline{b_{i j l}} g_{j}\left(x_{j}\left(t-\sigma_{i j l}(t)\right)\right) g_{l}\left(x_{l}\left(t-v_{i j l}(t)\right)\right)-\overline{I_{i}} \mid \\
\geq & \sum_{i=1}^{n} \overline{c_{i}}\left|x_{i}(t)\right|-\sum_{i=1}^{n} \sum_{j=1}^{n}\left|\overline{a_{i j}}\right| M_{j} \\
& -\sum_{i=1}^{n} \sum_{j=1}^{n} \sum_{l=1}^{n} \overline{b_{i j l}} N_{j} N_{l}-\sum_{i=1}^{n}\left|\overline{I_{i}}\right| \\
\geq & \sum_{i=1}^{n}\left(\overline{c_{i}}\left|x_{i}(t)\right|\right) \\
& -\sum_{i=1}^{n}\left(\left|\overline{I_{i}}\right|+\sum_{j=1}^{n}\left|\overline{a_{i j}}\right| M_{j}+\sum_{j=1}^{n} \sum_{l=1}^{n} \overline{b_{i j l}} N_{j} N_{l}\right) \\
\geq & \min _{1 \leq i \leq n}\left(\overline{c_{i}}\right) \sum_{i=1}^{n}\left|x_{i}(t)\right| \\
& -n \max _{1 \leq i \leq n}\left(\left|\overline{I_{i}}\right|+\sum_{j=1}^{n}\left|\overline{a_{i j}}\right| M_{j}+\sum_{j=1}^{n} \bar{l} \sum_{l=1}^{n}\left|\overline{b_{i j l}}\right| N_{j} N_{l}\right) \\
> & 0 .
\end{aligned}
$$

Consequently,

$$
Q N\left(x_{1}(t), x_{2}(t), \ldots, x_{n}(t)\right)^{T} \neq(0,0, \ldots, 0)^{T}
$$


for $\left(x_{1}(t), x_{2}(t), \ldots, x_{n}(t)\right)^{T} \in \partial \Omega \bigcap \operatorname{Ker} L$. This satisfies condition (2) of Theorem 2.8. Define $\Psi: \operatorname{Ker} L \times[0,1] \rightarrow X$ by

$$
\Psi\left(x_{1}, x_{2}, \ldots, x_{n}, \mu\right)=-\mu\left(x_{1}, x_{2}, \ldots, x_{n}\right)^{T}+(1-\mu) Q N\left(x_{1}(t), x_{2}(t), \ldots, x_{n}(t)\right)^{T}
$$

When $\left(x_{1}(t), x_{2}(t), \ldots, x_{n}(t)\right)^{T} \in \partial \Omega \bigcap \operatorname{Ker} L,\left(x_{1}, x_{2}, \ldots, x_{n}\right)^{T}$ is a constant vector in $\mathbb{R}^{n}$ with $\sum_{i=1}^{n}\left|x_{i}\right|=H^{*}$, we easily have $\Psi\left(x_{1}, x_{2}, \ldots, x_{n}, \mu\right) \neq(0,0, \ldots, 0)^{T}$. Therefore

$$
\begin{aligned}
\operatorname{deg} & \left(Q N\left(x_{1}(t), x_{2}(t), \ldots, x_{n}(t)\right)^{T}, \Omega \bigcap \operatorname{Ker} L,(0,0, \ldots, 0)^{T}\right) \\
& =\operatorname{deg}\left(\left(-x_{1}(t),-x_{2}(t), \ldots,-x_{n}(t)^{T}, \Omega \bigcap \operatorname{Ker} L,(0,0, \ldots, 0)^{T}\right) \neq 0 .\right.
\end{aligned}
$$

Condition (3) of Theorem 2.8 is also satisfied. Thus, by Theorem 2.8 we can obtain that $L x=$ $N x$ has at least one solution in $X$. That is, system (1.2) has at least one $\omega$-periodic solution. The proof is complete.

\section{Global Exponential Stability of Periodic Solution}

In this section, we will construct suitable Lyapunov functions to study the global exponential stability of the periodic solution of (1.2) on time scales. So first we will introduce some definitions.

Definition 4.1 (see [12]). A function $f$ from $\mathbb{T}$ to $\mathbb{R}$ is positively regressive if $1+\mu(t) f(t)>0$ for every $t \in \mathbb{T}$.

Denote $\mathfrak{R}^{+}$is the set of positively regressive functions from $\mathbb{T}$ to $\mathbb{R}$, and denote $\mathbb{T}^{+}=$ $[0,+\infty) \cap \mathbb{T}$.

Definition 4.2. The periodic solution $x^{*}(t)$ of system (1.2) is said to be exponentially stable if there exists a positive constant $\alpha$ with $-\alpha \in \mathfrak{R}^{+}$such that for every $\delta \in \mathbb{T}$, there exists $N=N(\delta) \geq 1$ such that the solution $x(t)$ of $(1.2)$ through $(\delta, \varphi(\delta))$ satisfies $\left\|x(t)-x^{*}(t)\right\| \leq$ $N\left\|\varphi(\delta)-x^{*}(\delta)\right\| e_{-\alpha}(t, \delta), t \in \mathbb{T}^{+}$where $\left\|\varphi(\delta)-x^{*}(\delta)\right\|=\sum_{i=1}^{n} \max _{\delta \in[-\theta, 0] \cap \mathbb{T}}\left|\varphi_{i}(\delta)-x_{i}^{*}(\delta)\right|$.

Theorem 4.3. Assume that $\left(H_{1}\right)-\left(H_{3}\right)$ hold. Suppose further that there exists $n$ positive constants $\epsilon_{i}>0, i=1,2, \ldots, n$ such that

$\left(H_{4}\right)$

$$
-\epsilon_{i} C_{i}^{-}+\sum_{j=1}^{n} a_{i j}^{+} M_{j} \epsilon_{j}+\sum_{j=1}^{n} \sum_{l=1}^{n} b_{i j l}^{+}\left(H_{j} N_{l} \epsilon_{j}+H_{l} N_{j} \epsilon_{l}\right)<0, \quad i=1,2, \ldots, n,
$$

then the w-periodic solution of system (1.2) is globally exponentially stable. 
Proof. According to Theorem 3.1, we know that (1.2) has an $\omega$-periodic solution $x^{*}(t)=$ $\left(x_{1}^{*}(t), x_{2}^{*}(t), \ldots, x_{n}^{*}(t)\right)^{T}$. Suppose that $x(t)=\left(x_{1}(t), x_{2}(t), \ldots, x_{n}(t)\right)^{T}$ is an arbitrary solution of (1.2). Then it follows from system (1.2) that

$$
\begin{aligned}
\left(x_{i}(t)-x_{i}^{*}(t)\right)^{\Delta}= & -c_{i}(t)\left(x_{i}(t)-x_{i}^{*}(t)\right)+\sum_{j=1}^{n} a_{i j}(t)\left(f_{j}\left(x_{j}\left(t-\gamma_{i j}(t)\right)\right)-f_{j}^{*}\left(x_{j}\left(t-\gamma_{i j}(t)\right)\right)\right) \\
& +\sum_{j=1}^{n} \sum_{l=1}^{n} b_{i j l}(t)\left(g_{j}\left(x_{j}\left(t-\sigma_{i j l}(t)\right)\right) g_{l}\left(x_{l}\left(t-v_{i j l}(t)\right)\right)\right) \\
& -g_{j}\left(x_{j}^{*}\left(t-\sigma_{i j l}(t)\right)\right) g_{l}\left(x_{l}^{*}\left(t-v_{i j l}(t)\right)\right), \quad i=1,2, \ldots, n,
\end{aligned}
$$

with initial values given by

$$
x_{i}(s)=\varphi_{i}(s), \quad s \in[-\theta, 0] \bigcap \mathbb{T}, i=1,2, \ldots, n,
$$

where $\theta$ is defined as in(1.3). If $\left(H_{4}\right)$ holds, it can always find a small enough constant $\alpha>0$ satisfying $\forall t \in \mathbb{T}, 1-\mu(t) \alpha>0$, namely, $-\alpha \in \mathfrak{R}^{+}$such that

$$
\begin{aligned}
\left(-c_{i}^{-}\right. & +\alpha) \epsilon_{i}+\sum_{j=1}^{n} a_{i j}^{+} M_{j} e_{\alpha}\left(t, t-\gamma_{i j}(t)\right) \epsilon_{j} \\
& +\sum_{j=1}^{n} \sum_{l=1}^{n} b_{i j l}^{+}\left[H_{j} N_{l} \epsilon_{j} e_{\alpha}\left(t, t-\sigma_{i j l}(t)\right)+H_{l} N_{j} \epsilon_{l} e_{\alpha}\left(t, t-v_{i j l}(t)\right)\right]<0, \quad i=1,2, \ldots, n
\end{aligned}
$$

We define a Lyapunov function $H=\left(h_{1}, h_{2}, \ldots, h_{n}\right)^{T}$ by $h_{i}=e_{\alpha}(t, \delta)\left|x_{i}(t)-x_{i}^{*}(t)\right|, \delta \in$ $[-\theta, 0] \cap \mathbb{T}, i=1,2, \ldots, n$. In view of $(4.2)$, we obtain

$$
\begin{aligned}
{\left[h_{i}^{\Delta}(t)\right]^{+}=} & \alpha e_{\alpha}(t, \delta)\left|x_{i}(t)-x_{i}^{*}(t)\right|+e_{\alpha}(\sigma(t), \delta) \operatorname{sign}\left(x_{i}(t)-x_{i}^{*}(t)\right) \\
\times & \left\{-c_{i}(t)\left(x_{i}(t)-x_{i}^{*}(t)\right)\right. \\
& +\sum_{j=1}^{n} a_{i j}(t)\left(f_{j}\left(x_{j}\left(t-\gamma_{i j}(t)\right)\right)-f_{j}\left(x_{j}^{*}\left(t-\gamma_{i j}(t)\right)\right)\right) \\
& +\sum_{j=1}^{n} \sum_{l=1}^{n} b_{i j l}(t)\left(g_{j}\left(x_{j}\left(t-\sigma_{i j l}(t)\right)\right) g_{l}\left(x_{l}\left(t-v_{i j l}(t)\right)\right)\right) \\
& \left.-g_{j}\left(x_{j}^{*}\left(t-\sigma_{i j l}(t)\right)\right) g_{l}\left(x_{l}^{*}\left(t-v_{i j l}(t)\right)\right)\right\}
\end{aligned}
$$


Discrete Dynamics in Nature and Society

$$
\begin{aligned}
& \leq \alpha e_{\alpha}(t, \delta)\left|x_{i}(t)-x_{i}^{*}(t)\right|+e_{\alpha}(\sigma(t), \delta) \\
& \times\left\{-c_{i}(t)\left|x_{i}(t)-x_{i}^{*}(t)\right|+\sum_{j=1}^{n}\left|a_{i j}(t)\right|\left|x_{j}\left(t-\gamma_{i j}(t)\right)-x_{j}^{*}\left(t-\gamma_{i j}(t)\right)\right| M_{j}\right. \\
& +\sum_{j=1}^{n} \sum_{l=1}^{n}\left|b_{i j l}(t)\right|\left[H_{j} N_{l}\left|x_{j}\left(t-\sigma_{i j l}(t)\right)-x_{j}^{*}\left(t-\sigma_{i j l}(t)\right)\right|\right. \\
& \left.\left.+H_{l} N_{j}\left|x_{l}\left(t-v_{i j l}(t)\right)-x_{l}^{*}\left(t-v_{i j l}(t)\right)\right|\right]\right\} \\
& \leq e_{\alpha}(\sigma(t), \delta) \\
& \times\left\{\left(-c_{i}(t)+\alpha\right)\left|x_{i}(t)-x_{i}^{*}(t)\right|+\sum_{j=1}^{n}\left|a_{i j}(t)\right|\left|x_{j}\left(t-\gamma_{i j}(t)\right)-x_{j}^{*}\left(t-\gamma_{i j}(t)\right)\right| M_{j}\right. \\
& +\sum_{j=1}^{n} \sum_{l=1}^{n}\left|b_{i j l}(t)\right|\left[H_{j} N_{l}\left|x_{j}\left(t-\sigma_{i j l}(t)\right)-x_{j}^{*}\left(t-\sigma_{i j l}(t)\right)\right|\right. \\
& \left.\left.+H_{l} N_{j}\left|x_{l}\left(t-v_{i j l}(t)\right)-x_{l}^{*}\left(t-v_{i j l}(t)\right)\right|\right]\right\} \\
& \leq[1+\mu(t) \alpha]\left\{\left(-c_{i}^{-}+\alpha\right) h_{i}(t)+\sum_{j=1}^{n} a_{i j}^{+} M_{j} e_{\alpha}\left(t, t-\gamma_{i j}(t)\right) h_{j}\left(t-\gamma_{i j}(t)\right)\right. \\
& +\sum_{j=1}^{n} \sum_{l=1}^{n} b_{i j l}^{+} \times\left[H_{j} N_{l} e_{\alpha}\left(t, t-\sigma_{i j l}(t)\right) h_{j}\left(t-\sigma_{i j l}(t)\right)\right. \\
& \left.\left.+H_{l} N_{j} e_{\alpha}\left(t, t-v_{i j l}(t)\right) h_{l}\left(t-v_{i j l}(t)\right)\right]\right\}, \\
& i=1,2 \ldots, n \text {. }
\end{aligned}
$$

Defining the curve $\rho=\left\{\underline{\omega}(l): \underline{\omega}_{i}=\epsilon_{i} l, l>0, i=1,2, \ldots, n\right\}$ and the set $\Omega(\underline{\omega})=\{\underline{u}$ : $0 \leq \underline{u} \leq \underline{\omega}\}, S_{i}(\underline{\omega})=\left\{\underline{u} \in \Omega(\underline{\omega}): \underline{u}_{i}=\underline{\omega}\right\}, i=1,2 \ldots, n$. It is obvious that if $l<\underline{l}$, then $\Omega(\underline{\omega}(l)) \subset \Omega(\underline{\omega}(\underline{l}))$. We will prove that the zero solution of (4.2) is exponential stable, namely, there exists a constant $\beta>0$ such that

$$
\left\|x(t)-x^{*}(t)\right\| \leq N(\delta) e_{-\beta}(t, \delta)\left\|\varphi(t)-x^{*}(t)\right\|, \quad t \geq 0 .
$$

Let $\epsilon^{M}=\max _{1 \leq i \leq n}\left\{\epsilon_{i}\right\}, \epsilon^{m}=\min _{1 \leq i \leq n}\left\{\varepsilon_{i}\right\}, l_{0}=(1-\delta)\left|\varphi_{i}-x_{i}^{*}\right|_{0} / \epsilon^{m}$, where $-\delta \geq 0$ is a constant, $\left|\varphi_{i}-x_{i}^{*}\right|_{0}=\max _{1 \leq i \leq n}\left\{\max _{\delta \in[-\theta, 0] \cap \mathbb{T}}\left|\varphi_{i}(\delta)-x_{i}^{*}(\delta)\right|\right\}$. Then, $\left\{|H|:|H|=e_{\alpha}(t, \delta) \mid \varphi(\delta)-\right.$ $\left.x^{*}(\delta) \mid,-\theta \leq t \leq \delta \leq 0\right\} \subset \Omega\left(\underline{\omega}\left(l_{0}\right)\right)$, namely,

$$
\left|h_{i}(\delta)\right|=e_{\alpha}(t, \delta)\left|\varphi_{i}(\delta)-x_{i}^{*}(\delta)\right|<\epsilon_{i} l_{0}, \quad-\theta \leq \delta \leq 0, i=1,2, \ldots, n
$$


We can claim that $\left|h_{i}(t)\right|<\epsilon_{i} l_{0}$ for $t>0, i=1,2, \ldots, n$. If it is not true, then there exist some $i$ and $t_{1}\left(t_{i}>0\right)$ such that $\left|h_{i}\left(t_{1}\right)\right|=\epsilon_{i} l_{0},\left[h_{i}^{\Delta}\left(t_{1}\right)\right]^{+} \geq 0$ and $\left|h_{i}(t)\right| \leq \epsilon_{i} l_{0}$ for $-\theta \leq t \leq t_{1}, i=$ $1,2, \ldots, n$. However, from (4.4) and (4.5), we get

$$
\begin{aligned}
{\left[h_{i}^{\Delta}\left(t_{1}\right)\right]^{+} \leq[1+\mu(t) \alpha] } & {\left[\left(-c_{i}^{-}+\alpha\right) \epsilon_{i}+\sum_{j=1}^{n} a_{i j}^{+} M_{j} e_{\alpha}\left(t, t-\gamma_{i j}(t)\right) \epsilon_{j}\right.} \\
& \left.+\sum_{j=1}^{n} \sum_{l=1}^{n} b_{i j l}^{+}\left[H_{j} N_{l} e_{\alpha}\left(t, t-\sigma_{i j l}(t)\right) \epsilon_{j}+H_{l} N_{j} e_{\alpha}\left(t, t-v_{i j l}(t)\right) \epsilon_{l}\right]\right] l_{0}<0
\end{aligned}
$$

for $t>0, i=1,2, \ldots, n$, this is a contradiction. So $\left|h_{i}(t)\right|<\epsilon_{i} l_{0}$, for $t \geq 0$. Also

$$
\left|x_{i}(t)-x_{i}^{*}(t)\right|<e_{\Theta \alpha}(t, \delta) \epsilon_{i} l_{0} \leq \frac{1}{\epsilon^{m}} e_{\Theta \alpha}(t, \delta) \epsilon_{i}(1-\delta)\left|\varphi_{i}-x_{i}^{*}\right|_{0^{\prime}} \quad i=1,2, \ldots, n, t \geq 0,
$$

which means that

$$
\left\|x(t)-x^{*}(t)\right\| \leq \frac{1}{\epsilon^{m}} e_{\Theta \alpha}(t, \delta) \epsilon^{M}(1-\delta)\left\|\varphi(\delta)-x^{*}(\delta)\right\| .
$$

Denote $-\beta=\Theta \alpha=-\alpha /(1+\mu \alpha) \in \mathfrak{R}^{+}, N=N(\delta)=\left(\epsilon^{M} / \epsilon^{m}\right)(1-\delta)>1$, in view of (4.10), we have

$$
\left\|x(t)-x^{*}(t)\right\| \leq N e_{-\beta}(t, \delta)\left\|\varphi(s)-x^{*}(s)\right\| .
$$

From Definition 4.2, the periodic solution of system (1.2) is globally exponentially stable. The proof is complete.

\section{An Example}

Let $\mathbb{T}=\mathbb{R}$, in this case, $x^{\Delta}(t)=d x(t) / d t$. Consider the following equation

$$
\begin{aligned}
\frac{d x_{i}(t)}{d t}= & -c_{i}(t) x_{i}(t)+\sum_{j=1}^{2} a_{i j}(t) f_{j}\left(x_{j}\left(t-\gamma_{i j}(t)\right)\right) \\
& +\sum_{j=1}^{2} \sum_{l=1}^{2} b_{i j l}(t) g_{j}\left(x_{j}\left(t-\sigma_{i j l}(t)\right)\right) g_{l}\left(x_{l}\left(t-v_{i j l}(t)\right)\right)+I_{i}(t), \quad i=1,2,
\end{aligned}
$$


where

$$
\begin{gathered}
f_{1}\left(x_{1}\right)=\sin \left(\frac{1}{2^{1 / 2}} x_{1}\right), \quad f_{2}\left(x_{2}\right)=\sin \left(\frac{1}{2^{3 / 2}} x_{2}\right), \\
g_{1}\left(x_{1}\right)=\arctan \left(\frac{1}{2^{1 / 2}} x_{1}\right), \quad g_{2}\left(x_{2}\right)=\arctan \left(\frac{1}{2^{3 / 2}} x_{2}\right) .
\end{gathered}
$$

Obviously, $f_{i}\left(x_{i}\right), g_{i}\left(x_{i}\right)(i=1,2)$ satisfy $\left(H_{1}\right)$ and $\left(H_{2}\right)$, and

$$
H_{1}=H_{2}=M_{1}=M_{2}=1, \quad N_{1}=N_{2}=\frac{\pi}{2} .
$$

Take

$$
\begin{gathered}
a_{11}(t)=1+\cos (2 \pi t), \quad a_{12}(t)=2+\cos (2 \pi t), \quad a_{21}(t)=2+\cos (2 \pi t), \quad a_{22}(t)=3+\cos (2 \pi t), \\
c_{1}(t)=20+5 \sin (2 \pi t), \quad c_{2}(t)=33+16 \sin (2 \pi t), \quad I_{1}(t)=1+\sin (2 \pi t), \quad I_{2}(t)=1+\cos (2 \pi t), \\
b_{111}(t)=b_{222}(t)=\frac{1}{4}+\frac{1}{4} \sin (2 \pi t), \quad b_{112}(t)=b_{212}(t)=\frac{1}{3}+\frac{1}{3} \cos (2 \pi t), \\
b_{121}(t)=b_{221}(t)=\frac{1}{5}+\frac{1}{5} \cos (2 \pi t), \quad b_{122}(t)=b_{211}(t)=\frac{1}{6}+\frac{1}{6} \sin (2 \pi t) .
\end{gathered}
$$

One can verifies that $\left(H_{1}\right)$ is satisfied, and $\omega=1, c_{1}^{-}=15, c_{2}^{-}=17, a_{11}^{+}=2, a_{12}^{+}=3, a_{21}^{+}=$ $3, a_{22}^{+}=4, b_{111}^{+}=b_{222}^{+}=1 / 2, b_{112}^{+}=b_{212}^{+}=2 / 3, b_{121}^{+}=b_{221}^{+}=2 / 5, b_{122}^{+}=b_{211}^{+}=1 / 3$, so, if we take $\epsilon_{1}=\epsilon_{2}=1$, we can obtain

$$
\begin{aligned}
& -\epsilon_{1} c_{1}^{-}+\sum_{j=1}^{2} a_{1 j}^{+} M_{j} \epsilon_{j}+\sum_{j=1}^{2} \sum_{l=1}^{2} b_{1 j l}^{+}\left(H_{j} N_{l} \epsilon_{j}+H_{l} N_{j} \epsilon_{l}\right)=-15+5+\frac{19}{10} \pi<0, \\
& -\epsilon_{2} C_{2}^{-}+\sum_{j=1}^{2} a_{2 j}^{+} M_{j} \epsilon_{j}+\sum_{j=1}^{2} \sum_{l=1}^{2} b_{2 j l}^{+}\left(H_{j} N_{l} \epsilon_{j}+H_{l} N_{j} \epsilon_{l}\right)=-17+7+\frac{19}{10} \pi<0 .
\end{aligned}
$$

Condition (4.2) is satisfied. From Theorem 3.1 and 4.3, we know that (5.1) has at least one 1-periodic solution and this solution is exponential stability.

\section{Conclusion}

Sufficient conditions are derived to guarantee the stability and existence of periodic solutions for a class of delayed high-order Hopfield neural networks on time scales. To the best of our knowledge, the results presented here have been not appeared in the related literature. In fact, both continuous and discrete systems, are very important in implementing and applications. But it is troublesome to study the existence and stability of periodic solutions for continuous and discrete systems respectively. Therefore, it is meaningful to study that on time scales which can unify the continuous and discrete situations. Also, our methods used in this paper may be applied to some other systems. 


\section{Acknowledgments}

This work is supported by the National Natural Sciences Foundation of People's Republic of China under Grant 1097118.

\section{References}

[1] Z. Wang, J. Fang, and X. Liu, "Global stability of stochastic high-order neural networks with discrete and distributed delays," Chaos, Solitons E Fractals, vol. 36, no. 2, pp. 388-396, 2008.

[2] B. Xiao and H. Meng, "Existence and exponential stability of positive almost periodic solutions for high-order Hopfield neural networks," Applied Mathematical Modelling, vol. 33, no. 1, pp. 532-542, 2009.

[3] X. Yi, J. Shao, Y. Yu, and B. Xiao, "New convergence behavior of high-order Hopfield neural networks with time-varying coefficients," Journal of Computational and Applied Mathematics, vol. 219, no. 1, pp. 216-222, 2008.

[4] B. Xu, Q. Wang, and X. Liao, "Stability analysis of high-order Hopfield type neural networks with uncertainty," Neurocomputing, vol. 71, no. 4-6, pp. 508-512, 2008.

[5] X.-Y. Lou and B.-T. Cui, "Novel global stability criteria for high-order Hopfield-type neural networks with time-varying delays," Journal of Mathematical Analysis and Applications, vol. 330, no. 1, pp. $144-$ $158,2007$.

[6] B. Xu, X. Liu, and X. Liao, "Global exponential stability of high order Hopfield type neural networks," Applied Mathematics and Computation, vol. 174, no. 1, pp. 98-116, 2006.

[7] B. Xiao and H. Meng, "Existence and exponential stability of positive almost periodic solutions for high-order Hopfield neural networks," Applied Mathematical Modelling, vol. 33, no. 1, pp. 532-542, 2009.

[8] B. Liu and L. Huang, "Existence and exponential stability of periodic solutions for a class of CohenGrossberg neural networks with time-varying delays," Chaos, Solitons \& Fractals, vol. 32, no. 2, pp. $617-627,2007$.

[9] F. Zhang and Y. Li, "Almost periodic solutions for higher-order Hopfield neural networks without bounded activation functions," Electronic Journal of Differential Equations, vol. 2007, no. 99, pp. 1-10, 2007.

[10] M. Bohner and A. Peterson, Dynamic Equations on Time Scales: An Introduction with Applications, Birkhäuser, Boston, Mass, USA, 2001.

[11] M. Bohner and A. Peterson, Eds., Advances in Dynamic Equations on Time Scales, Birkhäuser, Boston, Mass, USA, 2003.

[12] S. Hilger, "Analysis on measure chains-a unified approach to continuous and discrete calculus," Results in Mathematics, vol. 18, no. 1-2, pp. 18-56, 1990.

[13] M. Bohner, M. Fan, and J. Zhang, "Existence of periodic solutions in predator-prey and competition dynamic systems," Nonlinear Analysis: Real World Applications, vol. 7, no. 5, pp. 1193-1204, 2006.

[14] F.-H. Wong, C.-C. Yeh, S.-L. Yu, and C.-H. Hong, "Young's inequality and related results on time scales," Applied Mathematics Letters, vol. 18, no. 9, pp. 983-988, 2005.

[15] J. Mawhin, Topological Degree Methods in Nonlinear Boundary Value Problems, vol. 40 of CBMS Regional Conference Series in Mathematics, American Mathematical Society, Providence, RI, USA, 1979. 


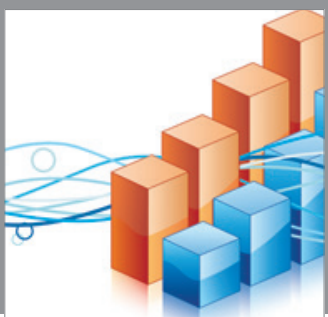

Advances in

Operations Research

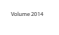

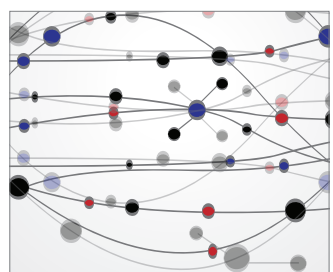

\section{The Scientific} World Journal
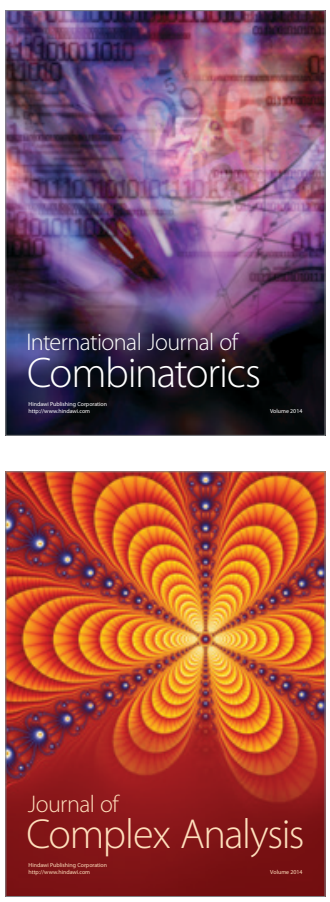

International Journal of

Mathematics and

Mathematical

Sciences
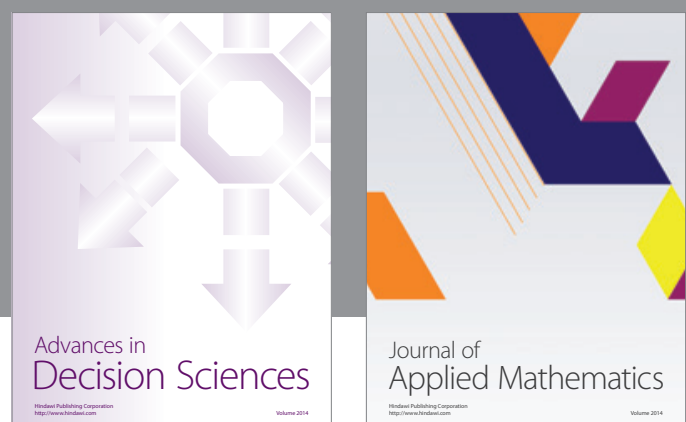

Journal of

Applied Mathematics
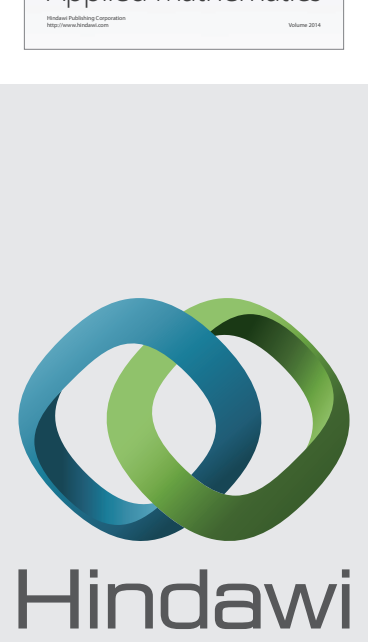

Submit your manuscripts at http://www.hindawi.com
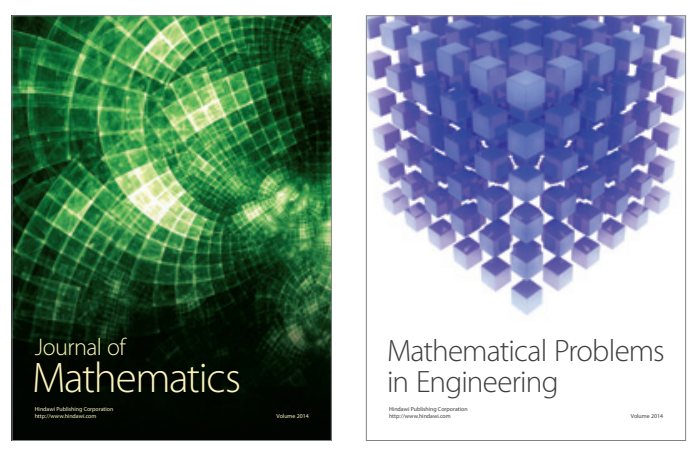

Mathematical Problems in Engineering
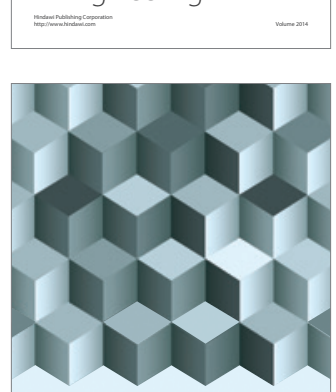

Journal of

Function Spaces
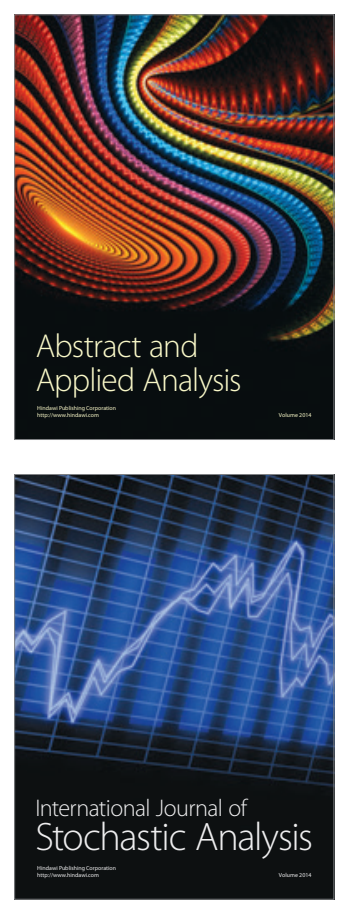

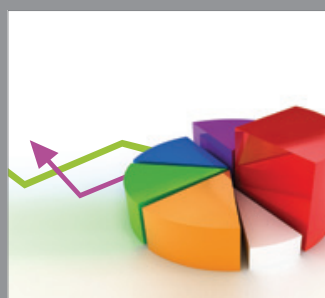

ournal of

Probability and Statistics

Promensencen
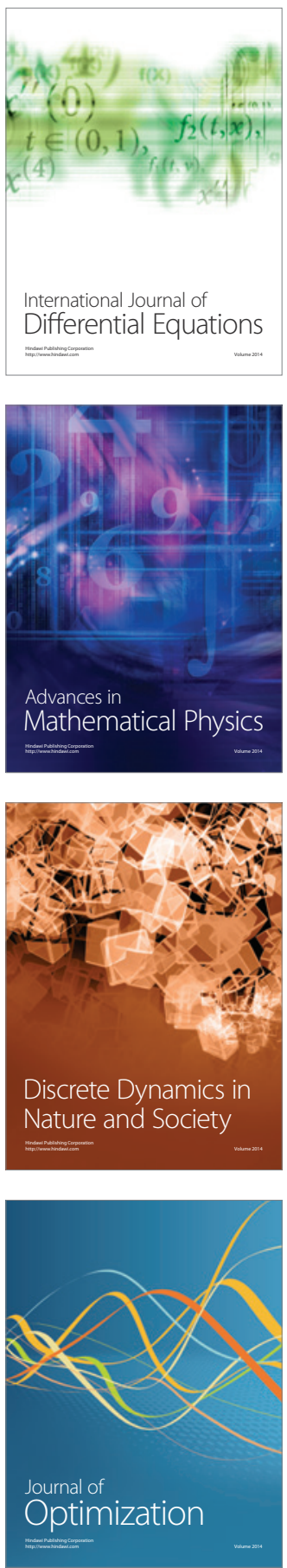